\title{
Morphological Characteristics of Drug-loaded Nanoparticles based on Microscope Images Analysis System
}

\author{
Bohua Feng, \\ College of Medical Information Engineering \\ Guangdong Pharmaceutical University \\ Guangzhou, China \\ tfengbh@163.com \\ Liufen Peng \\ College of Medical Information Engineering \\ Guangdong Pharmaceutical University \\ Guangzhou, China
}

\author{
Siyuan Mo \\ College of Medical Information Engineering \\ Guangdong Pharmaceutical University \\ Guangzhou, China \\ Huoyan $\mathrm{Xu}$ \\ College of Medical Information Engineering \\ Guangdong Pharmaceutical University \\ Guangzhou, China
}

\begin{abstract}
Microscope images analysis of drug carriers was a widely used method for those nanomaterials. Usually manual measuring and analysis of microscope images were subject to errors, and bad stability of results. Nanoparticles microscope images analysis method was combined with computer image processing technique in this research. A new nanoparticle Rot/CMC-g-RA was prepared with chitosan derivate drug carrier CMC-g-RA and botanical drug rotenone (Rot), and a processing and measuring system for microscope images of those drug-loaded nanoparticles was prepared by Visual $\mathrm{C}++\mathbf{6 . 0}$. The result showed characteristics measured by the system such as nanoparticles, particle size, area and roundness of Rot/CMC-gRA were close to that measured by dynamic light scattering (DLS) method. And a fast, precise and automatic method for measuring and statistic nanoparticles morphometric parameters was developed.
\end{abstract}

Keywords-component; Nanoparticle; Microscope Image Analysis; Morphological Characteristics

\section{INTRODUCTION}

Nanotechnology research and industrial products demanded strict quality control during its rapid development, in which more nanoparticles characteristics including morphological characteristics were required to be investigated with a higher precision. The surface morphology of nanoparticles could be observed and characterized by transmission electron microscope (TEM) and scanning electron microscope (SEM). And the nanoscale microscope images obtained by above instruments were usually characterized manually during the thirty years' development, which easily leaded to inefficient processing and errors[1]. Thus, a fast, reliable, and automatic new technique was required. The scope of this paper is to measure the morphological characteristics of Rot/CMC-g-RA nanoparticle which was prepared with chitosan derivate drug carrier CMC-g-RA and botanical drug rotenone (Rot), and it was carried out by SEM and a microscope images analysis

This project is supported by China National Natural Science Foundation (30771419 and 30971915). system (MIAS). MIAS was composed by four modules, in which microscope images processing and analysis were designed as two core modules. The former contributed to functions for examples image enhancement, point operation, setting the fill color, filling empty image, edge detection and image morphology. And the analysis module would achieve nanoparticles characteristics measuring including particle numbers, size, area and roundness of Rot/CMC-g-RA. The measuring results would also be compared with that obtained by dynamic light scattering (DLS) method.

\section{EXPERIMENTAL}

\section{A. Materials}

CMC-g-RA was prepared by polymers laboratory of deptment of materials science and engineering in Jinan university (Guangzhou, China). Rotenone was obtained from the Jiaxing chemistry factory $(98 \%$ pure, Fengshun of Guangdong, China). Acetone was obtained from Damao chemistry factory (A.R. Tianjin, China). All other reagents were analytical grade and were used directly without further purification. All solvents and water were redistilled freshly.

\section{B. Device}

Scanning electron microscope (SEM, XL-30E, Philips, Netherland), dynamic light scattering (DLS, BI-200SM, Brookhaven, USA), ZetaPALS (Brookhaven, USA), magnetic stirring apparatus (85-2, Xingchen Instruments, China), computer (G470, Lenovo, China) and MIAS software (developed by authors).

\section{Preparation of Rot/CMC-g-RA Nanoparticles Water Dispersion [2]}

The samples of CMC-g-RA were dissolved in deionized water $(\mathrm{pH}=6.8)$ to obtain the carrier solution $(10 \mathrm{mg} / \mathrm{ml})$. The 
Rot solution was then blended with the carrier solutions of given concentrations to form Rot/CMC-g-RA water dispersions under $200 \mathrm{rpm}$ stirring by magnetic stirring apparatus at room temperature for $30 \mathrm{~min}$, where the concentrations ratio of CMC-g-RA to Rot was 10.

\section{Characterization of Rot/ CMC-g-RA Nanoparticles [3]}

The nanoparticle morphological structure was demonstrated by SEM after dropping and drying the solutions onto base chips. The SEM photos were then processed and analyzed by MIAS to obtain size, area, roundness. The particle size, polydispersity index (PDI) were investigated with Zeta PALS.

\section{RESULTS AND DISCUSSION}

\section{A. Nanoparticles Morphological Structure}

SEM photo of Rot/CMC-g-RA particles was shown in Figure 1. These were smooth, spherical particles of sizes primarily less than $500 \mathrm{~nm}$, and they were well dispersed.

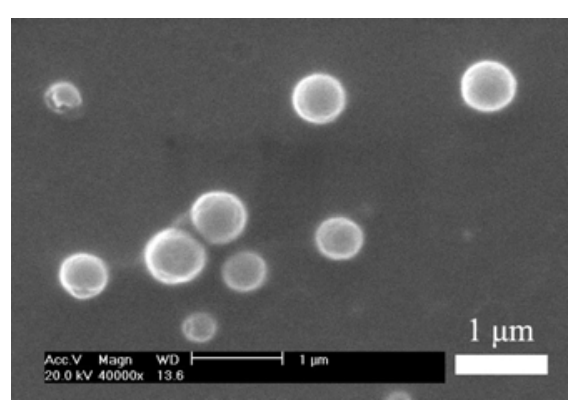

Figure 1. SEM photos of nanoparticles, $[\mathrm{CMC}-\mathrm{g}-\mathrm{RA}] /[\mathrm{Rot}]=10$.

Hydrophilic carboxymethyl trended toward the water phase while the hydrophobic $\mathrm{R}$ chain trended toward the oil phase to reduce surface tension, which formed shells of particles. Rot must have been effectively loaded into the carrier because the crystalline form of Rot was not readily observed.

\section{B. Nanoparticles Microscope Images Enhancement and Binarization}

The SEM photo's quality was usually low because of low contrast and high noise, which would affect image segmentation during processing. Therefore, median filtering technique was applied to removing image noise. A pixel with its coordinates $(\mathrm{x}, \mathrm{y})$ was set as a center point in the microscope image, around which pixels distributed within the range of $3 \times 3$ area were sorted in ascending order of gray levels. And the mid-value of those pixels gray levels was considered as the center pixel's gray level. This procedure was achieved by transferring original image pixels data to buffer, then affirmative mid-value of each pixel in buffer was copied to image to overwrite the original data. The nanoparticles in microscope image then became smooth in details with lower noise and separating from image background, which was contributed to further analysis.
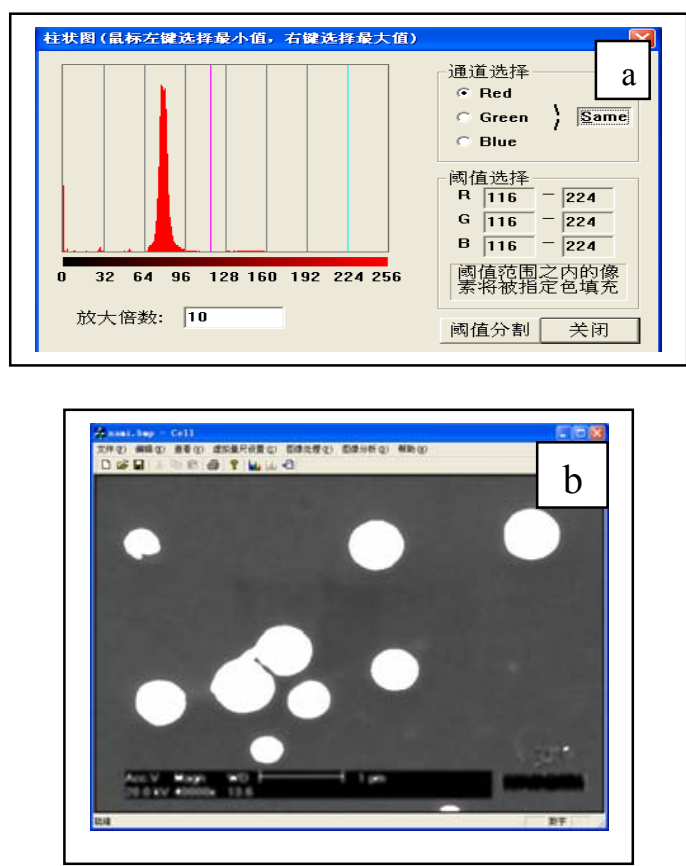

Figure 2. Selecting histogram thresholding(a), filling the empty regions(b).

Image point operation was implemented by histogram thresholding method. The distribution of gray levels in microscope image was shown in Figure 2a as a 2-dimension histogram, which horizontal ordinate referred to gray levels, and vertical coordinates represented gray levels frequency in image. The scope of gray levels threshold was easily adjusted in MIAS to achieve image binarization. And the pixels with gray levels within the threshold range were marked by MIAS. Non-marked and connected image regions were then found automaticly, and the smaller regions were filled by specified color as shown in Figure $2 b$, which empty small regions in original nanoparticles image (Figure 1) were connected. Closed areas were formed in nanoparitcles regions.

\section{Nanoparticles Edge Detection Algorithm}

Nanoparticles edges in the microscope image was investigated by means of Sobel operator, which could decrease noise and obtain accurate edge's direction. According to a pixel's gray level weighted difference from its adjacent pixels, the nanoparitlce's edge was detected as the value as the maximum was reached. Two $3 \times 3$ convolution kernels (odd number in both rows and columns) in Sobel operator sperately calcu-lated planar convolved with pixel gray level data, and the brightness difference approximations was obtained. The calculation process was shown in (1) to 3, where A represented original image, Gx and Gy separately standed for image edge detected in horizontal and vertical directions. Gray gradient value of single pixel were thus calculated in the two directions by (2). Furthermore, gray gradient direction angle $\theta$ in (3) was gained, where $\theta$ equaled to zero indicating a longitudinal edge of nanoparticle with higher brightness in the right side of its edge. 


$$
\begin{aligned}
& \mathbf{G}_{\mathbf{x}}=\left[\begin{array}{rrr}
-1 & 0 & +1 \\
-2 & 0 & +2 \\
-1 & 0 & +1
\end{array}\right] * A \text { and } \mathbf{G}_{\mathbf{y}}=\left[\begin{array}{ccc}
+1 & +2 & +1 \\
0 & 0 & 0 \\
-1 & -2 & -1
\end{array}\right] * A \\
& \mathbf{G}=\sqrt{\mathbf{G}_{\mathbf{x}}{ }^{2}+\mathbf{G}_{\mathbf{y}}{ }^{2}} \\
& \Theta=\arctan \left(\frac{\mathbf{G}_{\mathbf{y}}}{\mathbf{G}_{\mathbf{x}}}\right)
\end{aligned}
$$

Some nanoparticles edges were blurred in the microscope image. As Sobel operator method was implemented, noise was eliminated and pixels of large gradient values were removed. Additionally, many small meaningless points should not be considered as Rot/CMC-g-RA nanoparticles, corrosion and expansion functions were applied to remove these points in the binarized image (Figure 3a). All those processes above prepared a clear image compared to the original one, and the nanoparticles was specifically recognized eliminating the noise. And Figure $3 \mathrm{~b}$ indicated the effect that nanoparticles were correctly selected by high-light circles.

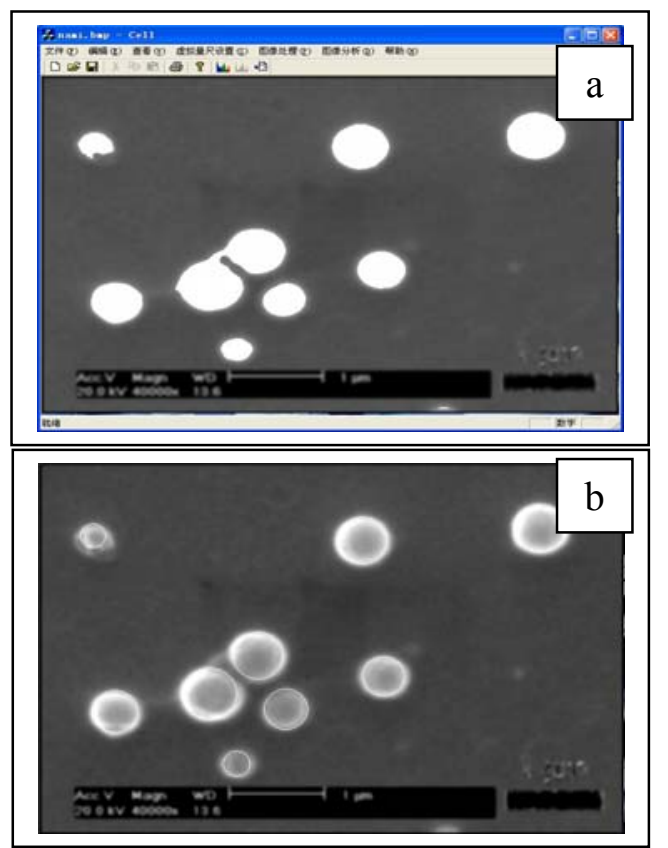

Figure 3. Image processed after Sobel algorithm and 3 corrosions (a), recognized nanoparticles(b)

\section{Microscope Image Automatic Analysis on Nanoparitlces Charateristics}

Image analysis module aimed to search nanoparticles center point and calculate numbers, average particles size, areas and perimeter. After many times corrosions and thinning, image of obvious nanoparticles had been shown in Figure 3b, where the highlight circle shapes made the center point searching and parameters calculation easier. The circle centers of each particles were marked and counted automatically, the particles diameters, areas, perimeter even roundness were calculated using geometric formulas by MIAS. Though pixel was considered as a suitable arithmatical unit in MIAS, international units(IU) were more useful for researchers respectively. So MIAS showed the calculation results in IU form as shown in Figure 4.

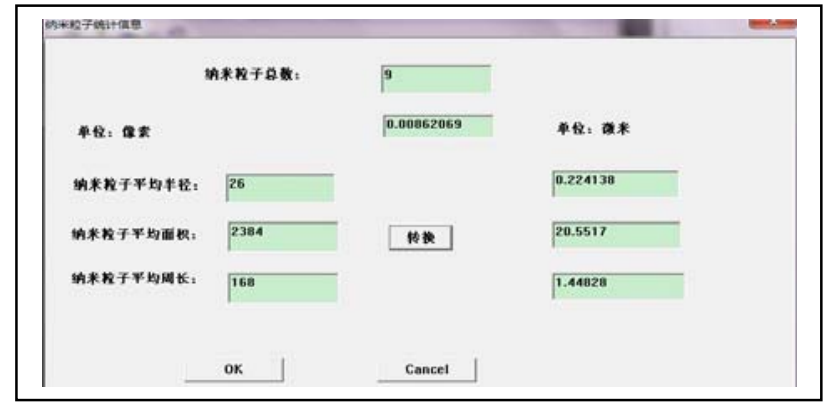

Figure 4. Calculation results of nanoparticles charateristics

\section{E. Measuring Accuracy of Nanoparticles Morphometric Parameters}

DLS was known as an accurate method for measuring nanoparticles particles size and PDI. Rot/CMC-g-RA nanoparticles with $[\mathrm{CMC}-\mathrm{g}-\mathrm{RA}] /[\mathrm{Rot}]=10$ demonstrated the particles size was $395.0 \pm 31.0 \mathrm{~nm}$ and PDI was 0.291 shown in Figure 5. It showed a moderate particles size, which was compatible with the SEM photo (Figure. 1).

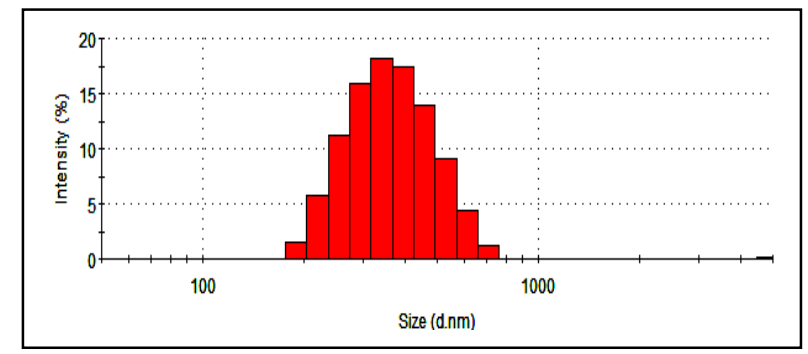

Figure 5. Particles size and PDI of Rot/CMC-g-RA nanoparticles by DLS

The particles size of Rot/CMC-g-RA achieved by MIAS was about $365.03 \pm 84.46 \mathrm{~nm}$ according to Figure 6. Moreover, MIAS also calculated that particles roundness was $0.78 \pm 0.02$, average area and perimeter were $2619.87 \pm 50 \mathrm{~nm}$ and $2.11 \times 105 \pm 7491.05 \mathrm{~nm}^{2}$ respectively, which demonstrated a similar result to that obtained by DLS method. The systematic error of MIAS could be derived from the procedure of preparing Rot/CMC-g-RA nanoparticles before taking SEM photo or DLS method. As dry samples were demanded in SEM, the nanoparticles size, roundness and volume were definitely different from that in water dispersion required in DLS.

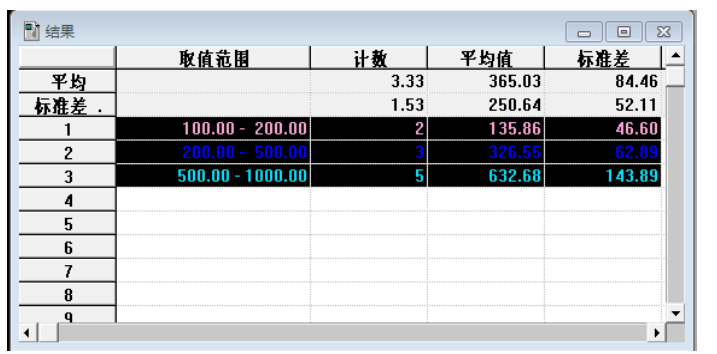

Figure 6. Particles size of Rot/CMC-g-RA nanoparticles by MIAS 


\section{CONCLUSIONS}

Rot/CMC-g-RA nanoparticles morphometric characteristics were measured and analyzed by microscope images analysis system. The result showed MIAS calculated and achieved a similar particle size to that using DLS. Addtionally, the particles roundness 0.78 was compatible with SEM photos. Therefore, MIAS indicated a prospect in characterizing nanoparticles morphometric parameters with fast and accuracy, which profited from computer image processing and SEM technology.

\section{REFERENCES}

[1] L. M. Wu and X. Y. Wang, "A new measurement method for submicrometer and nanometer particle size analysis-photon correlation spectroscopy," Shanghai Measurement and Testing, vol. 30, pp. 15-18, 2003.

[2] B. H. Feng and Z. Y. Zhang, "Rotenone/chitosan graft copolymer nanoparticle aqueous dispersion pesticide formulation," Chinese Journal of Pesticide Science, vol. 13, pp. 64-70, 2011.

[3] B. H. Feng and L. F. Peng, "Synthesis and characterization of carboxymethyl chitosan carrying rici-noleic functions as an emulsifier for azadirachtin," Carbohydrate Polymers, vol. 88, pp. 576-582, 2012.

[4] H. Li, "Edge detection technology of image processing," Agriculture \& Technology, vol. 30, pp. 163-165, 2010.

[5] J. Rosenman and Cullip, "High-performance computing in radiation cancer treatment," CRC Critical Reviews in BiomedicalEngineering, vol. 20, pp. 391-402, 1992. 